\title{
Ablation laser par laser UV impulsionnel : principale approche pour la synthèse controlée des nanostructures de silicium
}

\author{
L. Patrone, W. Marine, V.I. Safarov et M.L. Sentis ${ }^{1}$
}

Groupement Interdisciplinaire Ablation Laser et Applications, GPEC, UMR 6631 du CNRS, Faculté des Sciences de Luminy, Case 901, 13288 Marseille cedex 09, France

${ }^{1}$ Groupement Interdisciplinaire Ablation Laser et Applications, LP3, FRE 2165 du CNRS, Faculté des Sciences de Luminy, Case 901, 13288 Marseille cedex 09, France

\begin{abstract}
Résumé. Nous montrons que l'utilisation d'un laser UV impulsionnel (ArF*, $\lambda=193 \mathrm{~nm}$ ) est très bénéfique pour la synthèse de nano-agrégats de $\mathrm{Si}$ dans le régime d'ablation. L'éjection ionique de monomères $\mathrm{Si}^{+}$et de petits agrégats $\mathrm{Si}_{\mathrm{x}}{ }^{+}(\mathrm{x}=2-11)$ augmente fortement le taux de condensation dans la plume induite par laser UV. De plus, l'importante production d'ions non thermiques assure la stabilisation de la température de la surface irradiée permettant de contrôler la taille des nano-agrégats $(1-5 \mathrm{~nm})$ à l'aide d'un seul paramètre : la fluence laser. On peut alors changer la position spectrale de la bande de photoluminescence des nano-agrégats de Si du proche IR au proche UV par la sélection de la taille des nano-agrégats. Ces résultats sont en accord avec l'effet du confinement quantique.
\end{abstract}

La découverte d'une luminescence intense dans le visible, à température ambiante, à partir de nanostructures de silicium [1] a ouvert des perspectives très intéressantes vers la réalisation de systèmes optoélectroniques intégrés à base de silicium. Contrairement à la plupart des autres techniques utilisées, l'ablation laser possède l'avantage d'être chimiquement propre. Nous montrons que l'ablation par laser UV impulsionnel permet la synthèse de nano-agrégats de tailles faiblement dispersées et facilement maîtrisables par les conditions de préparation. Nous l'avons appliquée à l'élaboration de nano-agrégats de Si afin d'étudier leurs propriétés de photoluminescence (PL) en relation avec leur taille $(1-5 \mathrm{~nm})$.

Les nano-agrégats sont condensés dans la plume d'ablation induite par un laser excimère ArF* impulsionnel $\left(\lambda=193 \mathrm{~nm}, \tau_{\mathrm{p}}=15 \mathrm{~ns}\right.$ ) confinée par un gaz de couverture inerte (4 Torr d'He). Les dispositifs expérimentaux de synthèse et de caractérisation des nano-agrégats sont décrits dans [2]. Il existe un flux important de particules revenant vers la cible qui concerne la plus grande part des agrégats et permet d'en réaliser un dépôt sur le plan de la cible en rotation. Le taux d'agrégation est alors augmenté [3] grâce aux ions issus à la fois du processus d'ablation - désorption de la surface du matériau cible et de la fragmentation des nano-agrégats déposés sur la zone irradiée [4]. L'importante éjection d'ions non thermiques observée se traduit par la perte d'une partie de l'énergie transmise au matériau qui conduit à sa fusion puis à sa vaporisation. Il en résulte une augmentation du seuil dynamique de fusion avec la fluence laser [4]. L'énergie impliquée dans la vaporisation du matériau obtenue par la différence entre l'énergie d'irradiation et l'énergie au seuil dynamique de fusion est tracée en fonction de la fluence laser sur la Fig. 1a. Ces résultats mettent en évidence un régime de faibles fluences laser $\left(0,8-3,5 \mathrm{~J} / \mathrm{cm}^{2}\right)$ pour lequel l'énergie mise en jeu dans la vaporisation augmente peu. Ce régime est donc associé à une stabilisation de la température de la surface irradiée et, par conséquent, de la température initiale de la plume induite par laser. Il a été montré [5] que la température initiale et la densité initiale de la plume sont les deux paramètres régissant la taille des nano-agrégats condensés en phase gazeuse mais avec deux influences contraires. En particulier, à température constante, la taille des agrégats augmente avec la densité initiale, donc avec la fluence laser. Dans le régime de fluences $0,8-3,5 \mathrm{~J} / \mathrm{cm}^{2}$ on peut donc accroître la taille des nano-agrégats de façon contrôlée par l'augmentation de la fluence laser tout en conservant une dispersion de taille réduite.

Les distributions de taille des nano-agrégats de $\mathrm{Si}$ mesurées au centre des dépôts préparés à différentes fluences laser sont présentées sur la Fig. 1b. Comme on peut le voir, la taille moyenne des agrégats croît avec la fluence laser et passe d'environ 1,3 à $2,7 \mathrm{~nm}$ alors que la fluence laser est augmentée de 1 à $3 \mathrm{~J} / \mathrm{cm}^{2}$. On vérifie que, dans ce régime de faibles fluences où la température est stabilisée, la distribution de taille est étroite avec une largeur à mi hauteur de $\pm 0,2-0,3 \mathrm{~nm}$. Pour une fluence laser de $3,9 \mathrm{~J} / \mathrm{cm}^{2}$ qui marque le début du régime d'instabilité de la température, la distribution de 
taille devient plus large et se compose de deux maxima respectivement autour de $2,7 \mathrm{~nm}$ et de $5,1 \mathrm{~nm}$. Les spectres de PL obtenus à partir des nano-agrégats déposés sont composés d'une ou de plusieurs larges bandes et sont similaires à ceux généralement observés avec du silicium poreux ou des nano-agrégats de silicium $[1,6]$. Cependant, ils se distinguent par la possibilité de sélectionner la position spectrale de la bande de PL dans une large gamme couvrant le proche IR au proche UV, suivant les paramètres de dépôt. La Fig. 1c montre les spectres de PL mesurés au centre des films de nano-agrégats de Si déposés sous 4 Torr d'He pour des fluences variées de 1 à $3,9 \mathrm{~J} / \mathrm{cm}^{2}$. On peut voir que lorsque la fluence augmente, le maximum de la bande de PL est déplacé vers les plus faibles énergies de photons dans une région spectrale s'étendant de 3,5 à $1,7 \mathrm{eV}$, en relation avec l'augmentation correspondante de la taille des agrégats élaborés (voir Fig. 1b). Cela indique que la PL que nous observons à partir de nos échantillons est due au confinement des électrons et des trous dans les nano-agrégats de Si. L'absence de maxima de bandes de PL entre 2,5 et 3,5 eV s'explique par un modèle de formation de bande de PL que nous avons proposé [2] en bon accord avec les calculs existants sur la structure électronique du Si nanocristallin $[7,8]$.

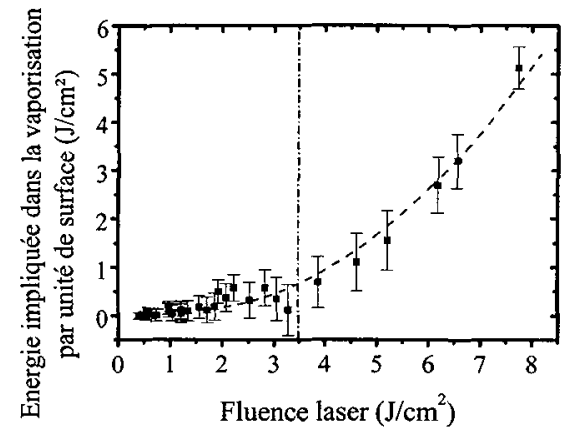

a)

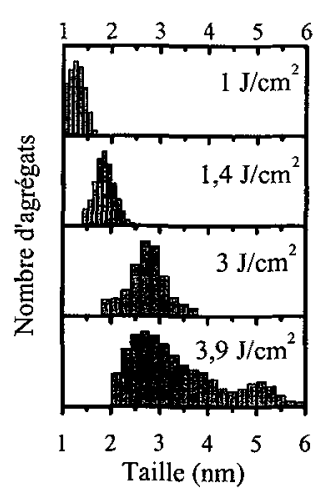

b)

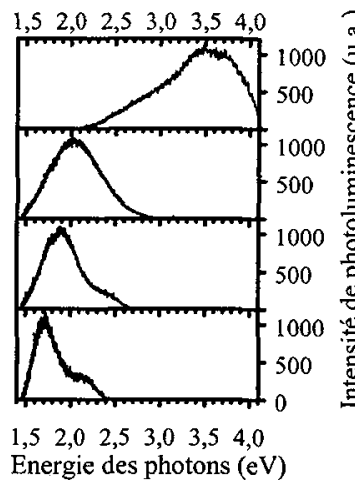

c)

Figure 1. a) Evolution de l'énergie par unité de surface impliquée dans la vaporisation (voir le texte) en fonction de la fluence laser d'irradiation. b) Distributions de taille des nano-agrégats de Si correspondant au centre des dépôts préparés à différentes fluences laser: $1 \mathrm{~J} / \mathrm{cm}^{2}, 1,4 \mathrm{~J} / \mathrm{cm}^{2}, 3 \mathrm{~J} / \mathrm{cm}^{2}$ et $3,9 \mathrm{~J} / \mathrm{cm}^{2}$. Les mesures de taille ont été réalisées par microscopie à force atomique sur des nano-agrégats de Si déposés sur un substrat de graphite HOPG. c) Spectres de PL correspondants des nano-agrégats de Si mesurés aux centres des dépôts. L'énergie des photons d'excitation est de 4,8 eV. L'intensité des spectres est normalisée.

En conclusion, les lasers UV impulsionnels sont très bien adaptés à la préparation de nano-agrégats de taille contrôlée avec une dispersion réduite grâce à la forte production d'ions non thermiques qu'ils engendrent. Ces derniers assurent la stabilisation de la température de la plume permettant le contrôle de la taille des agrégats par la fluence laser. En corrélation, on peut déplacer la bande de PL des nanoagrégats de Si dans un large domaine spectral étendu du proche UV au proche IR. La dépendance de la PL mesurée en fonction de la taille des agrégats est en accord avec l'hypothèse du confinement quantique.

\section{Références}

[1] L.T. Canham, Appl. Phys. Lett. 57, 1046 (1990).

[2] L. Patrone, D. Nelson, V.I. Safarov, M. Sentis, W. Marine, S. Giorgio, J. Appl. Phys. 87, 3829 (2000).

[3] C.T.R. Wilson in Nobel Lectures, Physics, 1922-1941 (1927), (Elsevier, Amsterdam, 1964-1970).

[4] L. Patrone, I. Ozerov, M. Sentis, W. Marine, «Interaction des photons UV avec le silicium massif et nanocristallin », this Proceedings.

[5] B.S. Luk'yanchuk, W. Marine, S.I. Anisimov, Laser Phys. 8, 1 (1998).

[6] E. Edelberg, S. Bergh, R. Naone, M. Hall, E.S. Aydil, J. Appl. Phys. 81, 2410 (1997).

[7] C. Delerue, G. Allan, M. Lannoo, Phys. Rev. B 48, 11024 (1993).

[8] L.-W. Wang, A. Zunger, J. Chem. Phys. 100, 2394 (1994). 